\title{
$Z^{\prime}$ generation with PYTHIA
}

\author{
Catalin Ciobanu, Thomas Junk, Gregory Veramendi \\ University of Illinois at Urbana-Champaign \\ Jedong Lee, Gilles De Lentdecker, Kevin McFarland \\ University of Rochester
}

Kaori Maeshima

Fermilab

November 23, 2008

\begin{abstract}
This document is intended as a guide for getting started with the $Z^{\prime}$ generation with PYTHIA[1]. Several different conventions used in literature are discussed, and the conversion among these is given. The $Z^{\prime}$ couplings to fermions are given for the sequential $Z^{\prime}$, the $Z^{\prime}$ model-lines of Ref. [2], and the popular E6 $Z^{\prime}$ models.
\end{abstract}

\section{Notations and Conventions}

The interaction of the $Z^{\prime}$ boson to Standard Model (SM) fermions $f$ can be generally written as:

$$
\mathcal{L}=g_{Z^{\prime}} \bar{f} \gamma^{\mu}\left(z_{f_{L}} P_{L}+z_{f_{R}} P_{R}\right) f Z_{\mu}^{\prime}
$$

where $g_{Z^{\prime}}$ is the $U(1)_{Z^{\prime}}$ gauge coupling, $z_{f_{L}}$ and $z_{f_{R}}$ are the left and right handed fermion charges, and $P_{L}=\left(1-\gamma^{5}\right) / 2$ and $P_{R}=\left(1+\gamma^{5}\right) / 2$ are the projectors for left and right- 

$\gamma^{5}$ term; we list below the main conventions used in literature:

$$
\begin{aligned}
& g_{Z^{\prime}} \bar{f} \gamma^{\mu}\left(z_{f_{L}} P_{L}+z_{f_{R}} P_{R}\right) f Z_{\mu}^{\prime} \\
& =g_{Z^{\prime}} \bar{f} \gamma^{\mu}\left(\frac{z_{f_{L}}+z_{f_{R}}}{2}-\frac{z_{f_{L}}-z_{f_{R}}}{2} \gamma^{5}\right) f Z_{\mu}^{\prime}= \\
& \equiv g_{Z^{\prime}} \bar{f} \gamma^{\mu}\left(C_{f_{V}}-C_{f_{A}} \gamma^{5}\right) f Z_{\mu}^{\prime} \\
& \equiv g_{Z^{\prime}} \bar{f} \gamma^{\mu} \frac{1}{2}\left(\mathcal{C}_{f_{\mathcal{V}}}-\mathcal{C}_{f_{\mathcal{A}}} \gamma^{5}\right) f Z_{\mu}^{\prime} \\
& \equiv-\bar{f} \gamma^{\mu}\left(C_{V_{Z^{\prime}, f}}+C_{A_{Z^{\prime}, f}} \gamma^{5}\right) f Z_{\mu}^{\prime} \\
& \equiv \frac{g}{4 \cos \theta_{W}} \bar{f} \gamma^{\mu}\left(C_{V}-C_{A} \gamma^{5}\right) f Z_{\mu}^{\prime}
\end{aligned}
$$$$
\text { CDDT }[2]
$$$$
\text { Halzen+Martin [3] }
$$$$
\text { Rosner87 [4] }
$$$$
\text { PYTHIA [1] }
$$

where $g=0.626$ is the $\mathrm{SU}(2)_{L}$ gauge coupling, and $\theta_{W}$ is the Weinberg angle. Note the slightly different notations used to identify the four conventions. The axial and vector couplings defined above can be expressed as:

$$
\begin{array}{lll}
C_{f_{V}}=\left(z_{f_{L}}+z_{f_{R}}\right) / 2 & C_{f_{A}}=\left(z_{f_{L}}-z_{f_{R}}\right) / 2 & \text { CDDT }[2] \\
\mathcal{C}_{f_{\mathcal{V}}}=z_{f_{L}}+z_{f_{R}} & \mathcal{C}_{f_{\mathcal{A}}}=z_{f_{L}}-z_{f_{R}} & \text { Halzen+Martin [3] } \\
C_{V_{Z^{\prime}, f}}=-g_{Z^{\prime}}\left(z_{f_{L}}+z_{f_{R}}\right) / 2 & C_{A_{Z^{\prime}, f}}=g_{Z^{\prime}}\left(z_{f_{L}}-z_{f_{R}}\right) / 2 & \text { Rosner87 [4] } \\
C_{V}=2 \cos \theta_{W}\left(z_{f_{L}}+z_{f_{R}}\right) g_{Z^{\prime}} / g & C_{A}=2 \cos \theta_{W}\left(z_{f_{L}}-z_{f_{R}}\right) g_{Z^{\prime}} / g & \text { PYTHIA [1] }
\end{array}
$$

From these conversion relations, it is now easy to express the input couplings to PYTHIA needed to implement various $Z^{\prime}$ models. As seen in Eqns. (4) and (5) (or Eqns. (8) and (9)), for the Rosner paper and PyтнiA cases the $g_{Z^{\prime}}$ is included in the $V$ and $A$ couplings. 


\begin{tabular}{lrrc}
\hline \hline$f$ & $Q_{f}$ & $T_{f}^{3}=\mathcal{C}_{f_{\mathcal{A}}}$ & \multicolumn{1}{c}{$\mathcal{C}_{f_{\mathcal{V}}}$} \\
\hline$\nu_{e}, \nu_{m u}, \ldots$ & 0 & $1 / 2$ & $1 / 2$ \\
$e^{-}, \mu^{-}, \ldots$ & -1 & $-1 / 2$ & $-1 / 2+2 \sin ^{2} \theta_{W}$ \\
$u, c, \ldots$ & $2 / 3$ & $1 / 2$ & $1 / 2-4 \sin ^{2} \theta_{W} / 3$ \\
$d, s, \ldots$ & $-1 / 3$ & $-1 / 2$ & $-1 / 2+2 \sin ^{2} \theta_{W} / 3$ \\
\hline \hline
\end{tabular}

Table 1: SM couplings, from page 301 of Halzen+Martin [3].

\begin{tabular}{|cc|cc|cc|cc|}
\hline \multicolumn{2}{|c|}{$d$} & \multicolumn{2}{|c|}{$u$} & \multicolumn{2}{c|}{$e$} & & $\nu_{e}$ \\
\hline$V$ & $A$ & $V$ & $A$ & $V$ & $A$ & $V$ \\
\hline PARU(121) & PARU(122) & PARU $(123)$ & PARU(124) & PARU(125) & PARU(126) & PARU(127) & PARU(128) \\
\hline$-1+\frac{4}{3} \sin ^{2} \theta_{W}$ & -1 & $1-\frac{8}{3} \sin ^{2} \theta_{W}$ & 1 & $-1+4 \sin ^{2} \theta_{W}$ & -1 & 1 \\
\hline-0.693 & -1 & 0.387 & 1 & -0.08 & -1 & 1 \\
\hline \hline
\end{tabular}

Table 2: SM couplings - PYTHIA implementation.

\section{PYTHIA Generation of Different $Z^{\prime}$ Models}

\subsection{Sequential $Z^{\prime}$}

The parameters available ${ }^{1}$ in PYTHIA to implement the couplings for a given $Z^{\prime}$ model are PARU(121)-PARU(128) for the $1^{\text {st }}$ generation quarks and leptons, PARJ(180)-PARJ(187) for the $2^{\text {nd }}$ generation, and PARJ(188)-PARJ(195) for the $3^{\text {rd }}$ generation, respectively. The default values are those of a sequential $Z^{\prime}$, which are the same as for the Standard Model $Z$ boson. In Halzen+Martin, these couplings are expressed as: $\mathcal{C}_{f_{\mathcal{V}}}=T_{f}^{3}-2 Q_{f} \sin ^{2} \theta_{W}$ and $\mathcal{C}_{f_{\mathcal{A}}}=T_{f}^{3}$ (see Table 1$)$

We can use Eqns. (7) and (9), plugging in the Standard Model $g_{Z^{\prime}}=g / \cos \theta_{W}$, to obtain the PYTHIA coefficients: $C_{V}=2 \mathcal{C}_{f_{\mathcal{V}}}$ and $C_{A}=2 \mathcal{C}_{f_{\mathcal{A}}}$, respectively. The exact values of the vector $(V)$ and axial $(A)$ couplings are given in the fourth row of Table 2 . If one uses the PYтHIA value $\sin ^{2} \theta_{W}=0.23$, one obtains the values listed in the fifth (last) row, which coincide with the defaults listed in the PүтніA manual. The couplings to the $2^{\text {nd }}$ and $3^{r d}$ families must be set to the same values, i.e. PARU(121)=PARJ(180)=PARJ(188), etc.

\subsection{CDDT model-lines}

In the CDDT paper [2], four general classes of $Z^{\prime}$ models (or model-lines) are discussed.

Table 3 presents the fermion charges for these models-lines. These charges are plugged in

\footnotetext{
${ }^{1}$ In addition to these, one can also set the $Z^{\prime}$ mass via PMAS(32) parameter, etc.
} 


\begin{tabular}{c|c|c|c|c|}
\hline \hline & $\mathrm{U}(1)_{B-x L}$ & $\mathrm{U}(1)_{q+x u}$ & $\mathrm{U}(1)_{10+x \overline{5}}$ & $\mathrm{U}(1)_{d-x u}$ \\
\hline$q_{L}$ & $1 / 3$ & $1 / 3$ & $1 / 3$ & 0 \\
$u_{R}$ & $1 / 3$ & $x / 3$ & $-1 / 3$ & $-x / 3$ \\
$d_{R}$ & $1 / 3$ & $(2-x) / 3$ & $-x / 3$ & $1 / 3$ \\
$l_{L}$ & $-x$ & -1 & $x / 3$ & $(-1+x) / 3$ \\
$e_{R}$ & $-x$ & $-(2+x) / 3$ & $-1 / 3$ & $x / 3$ \\
$\nu_{R}$ & 0 & 0 & 0 & 0 \\
\hline \hline
\end{tabular}

Table 3: The fermion gauge charges (adapted from Ref.[2]).

\begin{tabular}{|c|c|c|c|c|c|c|c|}
\hline \multicolumn{2}{|c|}{$d$} & \multicolumn{2}{|c|}{$u$} & \multicolumn{2}{|c|}{$e$} & \multicolumn{2}{|c|}{$\nu_{e}$} \\
\hline$V$ & $A$ & $V$ & $A$ & $V$ & $A$ & $V$ & $A$ \\
\hline PARU(121) & PARU(122) & PARU(123) & PARU(124) & PARU(125) & PARU(126) & PARU(127) & PARU(128) \\
\hline \multicolumn{8}{|c|}{$B-x L$ model-line } \\
\hline$\frac{4}{3} \gamma_{Z^{\prime}}$ & 0 & $\frac{4}{3} \gamma_{Z^{\prime}}$ & 0 & $-4 x \gamma_{Z^{\prime}}$ & 0 & $-2 x \gamma_{Z^{\prime}}$ & $-2 x \gamma_{Z^{\prime}}$ \\
\hline \multicolumn{8}{|c|}{$d-x u$ model-line } \\
\hline$\frac{2}{3} \gamma_{Z^{\prime}}$ & $-\frac{2}{3} \gamma_{Z^{\prime}}$ & $-\frac{2 x}{3} \gamma_{Z^{\prime}}$ & $\frac{2 x}{3} \gamma_{Z^{\prime}}$ & $\frac{2(2 x-1)}{3} \gamma_{Z^{\prime}}$ & $-\frac{2}{3} \gamma_{Z^{\prime}}$ & $\frac{2(x-1)}{3} \gamma_{Z^{\prime}}$ & $\frac{2(x-1)}{3} \gamma_{Z^{\prime}}$ \\
\hline \multicolumn{8}{|c|}{$q+x u$ model-line } \\
\hline$-\frac{2(x-3)}{3} \gamma_{Z^{\prime}}$ & $\frac{2(x-1)}{3} \gamma_{Z^{\prime}}$ & $\frac{2(x+1)}{3} \gamma_{Z^{\prime}}$ & $-\frac{2(x-1)}{3} \gamma_{Z^{\prime}}$ & $-\frac{2(x+5)}{3} \gamma_{Z^{\prime}}$ & $\frac{2(x-1)}{3} \gamma_{Z^{\prime}}$ & $-2 \gamma_{Z^{\prime}}$ & $-2 \gamma_{Z^{\prime}}$ \\
\hline \multicolumn{8}{|c|}{$10+x 5$ model-line } \\
\hline$-\frac{2(x-1)}{3} \gamma_{Z^{\prime}}$ & $\frac{2(x+1)}{3} \gamma_{Z^{\prime}}$ & 0 & $\frac{4}{3} \gamma_{Z^{\prime}}$ & $\frac{2(x-1)}{3} \gamma_{Z^{\prime}}$ & $\frac{2(x+1)}{3} \gamma_{Z^{\prime}}$ & $\frac{2 x}{3} \gamma_{Z^{\prime}}$ & $\frac{2 x}{3} \gamma_{Z^{\prime}}$ \\
\hline
\end{tabular}

Table 4: The four CDDT model-lines - PyтнiA implementation. The parameter $\gamma_{Z^{\prime}}$ is given by Eqn. (10), or $\gamma_{Z^{\prime}}=1.402 \cdot g_{Z^{\prime}}$ (using the PYTHIA $\sin ^{2} \theta_{W}=0.23$ and $g=0.626$ values).

Eqn. (9) to obtain the corresponding PyтHIA parameters, listed in Table 4. The following linear change of variable has been made:

$$
g_{Z^{\prime}}=\gamma_{Z^{\prime}} \cdot \frac{g}{\cos \theta_{W}} \quad \text { with } \quad \gamma_{Z^{\prime}} \equiv \frac{g_{Z^{\prime}}}{g} \cos \theta_{W}
$$

For example, for a $q+x u$ family $Z^{\prime}$ with $g_{Z^{\prime}}=0.1$ and $x=1.0$, we have PARU $(121)=$ $\operatorname{PARJ}(180)=\operatorname{PARJ}(188)=-2(1-3) \cdot 1.402 \cdot 0.1 / 3=0.187$, etc.

\section{$2.3 \quad$ E6 $Z$ 's}

In the E6 models, the SM structure is extended to include the $\mathrm{U}(1)_{\psi} \times \mathrm{U}(1)_{\chi}$ groups $[5]$. The gauge fields corresponding to these groups $Z_{\psi}^{\prime}$ and $Z_{\chi}^{\prime}$ can be massive, and are not 
true mass eigenstates since these states can mix. Let us define the mass eigenstates by:

$$
Z^{\prime}(\theta) \equiv Z_{\psi}^{\prime} \cos \theta+Z_{\chi}^{\prime} \sin \theta, \quad Z^{\prime \prime}(\theta) \equiv Z_{\psi}^{\prime} \sin \theta-Z_{\chi}^{\prime} \cos \theta
$$

with $\theta$ being a parameter dependent on the Higgs vev's and the gauge couplings $g_{\psi}$ and $g_{\chi}$ corresponding to $\mathrm{U}(1)_{\psi}$ and $\mathrm{U}(1)_{\chi}$. Different definitions of $\theta$ exist in the literature; however, this is irrelevant if one restricts the discussion only to $Z_{\psi}^{\prime}, Z_{\chi}^{\prime}, Z_{\eta}^{\prime}$, and $Z_{I}^{\prime}$ as it is customarily the case in experimental searches. In what follow we assume the $\theta$ definition from Ref. [4] (Rosner). As we will show, other definitions [2, 5] lead to the same results. To simplify the problem, we assume that $Z^{\prime \prime}(\theta)$ is heavy enough to decouple from the $Z$ and $Z^{\prime}(\theta)$, and will not be discussed further. The $Z^{\prime}(\theta)$ is light enough to mix with the standard model Z. According to Eqn. (3) of Ref. [4], we can write:

$$
g_{\theta}=\sqrt{\frac{5}{3}} g_{Z} \sin \theta_{W}=\sqrt{\frac{5}{3}} g \tan \theta_{W} \equiv \sqrt{\frac{5}{3}} \gamma
$$

where $g=0.626$ is the $\mathrm{SU}(2)_{L}$ gauge coupling. In this notation, and using Table I of Ref. [4], we can calculate the fermion couplings for the $Z^{\prime}(\theta)$, listed in Table 5. For this Table, to get the neutrino couplings we used the $e$ couplings and Eqn. (8) to calculate the $z_{\nu_{L}}$ charge $\left(z_{\nu_{L}}=z_{e_{L}}, z_{\nu_{R}}=0\right)$. To get the PYTHIA parameters, we used the relations from Eqns. (8) and (9).

Comparing to the $10+x \overline{5}$ results from the last row of Table 4, the following conversion relations can be written down:

$$
\begin{array}{ll}
\gamma_{Z^{\prime}}=\frac{\sqrt{10} \cos \theta+\sqrt{6} \sin \theta}{4} s, & \text { with } \quad s \equiv \sin \theta_{W} \\
x \gamma_{Z^{\prime}}=\frac{\sqrt{10} \cos \theta-3 \sqrt{6} \sin \theta}{4} s, & \text { with } \quad s \equiv \sin \theta_{W}
\end{array}
$$




\begin{tabular}{|c|c|c|c|c|c|c|}
\hline & $d$ & $u$ & & $\bar{e}$ & 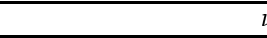 & \\
\hline \multicolumn{7}{|c|}{ Rosner convention } \\
\hline$-\frac{\sin \theta}{\sqrt{6}} \gamma$ & $\frac{\sqrt{5} \cos \theta-\sqrt{3} \sin \theta}{6 \sqrt{2}} \gamma$ & $\frac{\sqrt{5} \cos \theta+\sqrt{3} \sin \theta}{6 \sqrt{2}} \gamma$ & $\frac{\sin \theta}{\sqrt{6}} \gamma$ & $\frac{\sqrt{5} \cos \theta-\sqrt{3} \sin \theta}{6 \sqrt{2}} \gamma$ & $-\frac{\sqrt{5} \cos \theta-3 \sqrt{3} \sin \theta}{12 \sqrt{2}} \gamma$ & $\frac{\sqrt{5} \cos \theta-3 \sqrt{3} \sin \theta}{12 \sqrt{2}} \gamma$ \\
\hline PARU(121) & PARU(122) & PARU(124) & PARU(125) & PARU(126) & PARU(127) & $\begin{array}{l}\text { PARU(128) } \\
\text { PARU }\end{array}$ \\
\hline$\frac{4 \sin \theta}{\sqrt{6}} s$ & $\frac{\sqrt{10} \cos \theta-\sqrt{6} \sin \theta}{3} s$ & $\frac{\sqrt{10} \cos \theta+\sqrt{6} \sin \theta}{3} s$ & $-\frac{4 \sin \theta}{\sqrt{6}} s$ & $\frac{\sqrt{10} \cos \theta-\sqrt{6} \sin \theta}{3} s$ & $\frac{\sqrt{10} \cos \theta-3 \sqrt{6} \sin \theta}{6} s$ & $\frac{\sqrt{10} \cos \theta-3 \sqrt{6} \sin \theta}{6} s$ \\
\hline
\end{tabular}

Table 5: The general E6 fermion couplings as function of $\theta$, following Rosner's definition of $\theta$. We used the shorthand notations $\gamma \equiv g \tan \theta_{W}$, and $s \equiv \sin \theta_{W}$, respectively. To fit all values in the Table, we omitted the $C_{V_{Z^{\prime}, u}}$ column, as this parameter is zero; that is, $\operatorname{PARU}(123)=0$ for all E6 models.

The next step is to particularize the general $Z^{\prime}(\theta)$ to obtain the popular models. Table 6 lists the couplings for these models; the values were obtained simply by plugging each $\theta$ value in the expressions from Table 5. Given Eqns. (13)-(14), the same results from Table 6 can also be found if one particularizes the CDDT model-lines as follows [6]:

- $Z_{\psi}^{\prime}$ is obtained in the $10+x \overline{5}$ model-line (last row in Table 4), for $x=1$ and $\gamma_{Z^{\prime}}=$ $\sqrt{\frac{5}{8}} \sin \theta_{W}$

- $Z_{\chi}^{\prime}$ is obtained in the $10+x \overline{5}$ model-line (last row in Table 4 ), for $x=-3$ and $\gamma_{Z^{\prime}}=\sqrt{\frac{3}{8}} \sin \theta_{W}$

- $Z_{\eta}^{\prime}$ is obtained in the $10+x \overline{5}$ model-line (last row in Table 4 ), for $x=-1 / 2$ and $\gamma_{Z^{\prime}}=\sin \theta_{W}$

- $Z_{I}^{\prime}$ is obtained ${ }^{2}$ in the $d-x u$ model-line (eighth row in Table 4 ), for $x=0$ and $\gamma_{Z^{\prime}}=\frac{\sqrt{15}}{2} \sin \theta_{W}$

- $Z_{s q}^{\prime}$ is obtained in the $10+x \overline{5}$ model-line (last row in Table 4 ), for $x=-8$ and $\gamma_{Z^{\prime}}=\frac{1}{4} \sin \theta_{W}$

\footnotetext{
${ }^{2}$ This is also the special $10+x \overline{5}$ case: $\gamma_{Z^{\prime}} \rightarrow 0$, and $x \gamma_{Z^{\prime}}=-\frac{\sqrt{15}}{2} \sin \theta_{W}($ as $x \rightarrow \infty)$
} 


\begin{tabular}{|c|c|c|c|c|c|c|c|}
\hline \multicolumn{2}{|c|}{$d$} & \multicolumn{2}{|c|}{$u$} & \multicolumn{2}{|c|}{$\bar{e}$} & \multicolumn{2}{|c|}{$\nu_{e}$} \\
\hline$V$ & $A$ & $V$ & $A$ & & $A$ & $V$ & $A$ \\
\hline$\overline{\text { PARU(121) }}$ & PARU(122) & PARU(123) & PARU(124) & PARU(125) & PARU(126) & PARU(127) & $\begin{array}{l}\text { PARU(128) } \\
\text { PARU }\end{array}$ \\
\hline \multicolumn{8}{|c|}{$Z_{\psi}^{\prime} \quad$ corresponds to $\theta=0^{\circ}$} \\
\hline 0 & $\frac{\sqrt{10} s}{3}$ & 0 & $\frac{\sqrt{10} s}{3}$ & 0 & $\frac{\sqrt{10} s}{3}$ & $\frac{\sqrt{10} s}{6}$ & $\frac{\sqrt{10} s}{6}$ \\
\hline \multicolumn{8}{|c|}{$Z_{\chi}^{\prime} \quad$ corresponds to $\theta=90^{\circ}$} \\
\hline$\frac{2 \sqrt{6} s}{3}$ & $-\frac{\sqrt{6} s}{3}$ & 0 & $\frac{\sqrt{6} s}{3}$ & $-\frac{2 \sqrt{6} s}{3}$ & $-\frac{\sqrt{6} s}{3}$ & $-\frac{\sqrt{6} s}{2}$ & $-\frac{\sqrt{6} s}{2}$ \\
\hline \multicolumn{8}{|c|}{$Z_{\eta}^{\prime} \quad$ corresponds to $\theta=37.76^{\circ}$, i.e. $\sin \theta=\sqrt{3 / 8}$ and $\cos \theta=\sqrt{5 / 8}$} \\
\hline$s$ & $\frac{s}{3}$ & 0 & $\frac{4 s}{3}$ & $-s$ & $\frac{s}{3}$ & $-\frac{s}{3}$ & $-\frac{s}{3}$ \\
\hline \multicolumn{8}{|c|}{$Z_{I}^{\prime} \quad$ corresponds to $\theta=127.76^{\circ}$, i.e. $\sin \theta=\sqrt{5 / 8}$ and $\cos \theta=-\sqrt{3 / 8}$} \\
\hline$\frac{\sqrt{15} s}{3}$ & $-\frac{\sqrt{15} s}{3}$ & 0 & 0 & $-\frac{\sqrt{15} s}{3}$ & $-\frac{\sqrt{15} s}{3}$ & $-\frac{\sqrt{15} s}{3}$ & $-\frac{\sqrt{15} s}{3}$ \\
\hline \multicolumn{8}{|c|}{$Z_{s q}^{\prime} \quad$ corresponds to $\theta=113.28^{\circ}$, i.e. $\sin \theta=3 \sqrt{6} / 8$ and $\cos \theta=-\sqrt{10} / 8$} \\
\hline$\frac{3 s}{2}$ & $-\frac{7 s}{6}$ & 0 & $\frac{s}{3}$ & $-\frac{3 s}{2}$ & $-\frac{7 s}{6}$ & $-\frac{4 s}{3}$ & $-\frac{4 s}{3}$ \\
\hline \multicolumn{8}{|c|}{$Z_{N}^{\prime} \quad$ corresponds to $\theta=-14.48^{\circ}$, i.e. $\sin \theta=-1 / 4$ and $\cos \theta=\sqrt{15} / 4$} \\
\hline$-\frac{\sqrt{6} s}{6}$ & $\frac{\sqrt{6} s}{2}$ & 0 & $\frac{\sqrt{6} s}{3}$ & $\frac{\sqrt{6} s}{6}$ & $\frac{\sqrt{6} s}{2}$ & $\frac{\sqrt{6} s}{3}$ & $\frac{\sqrt{6} s}{3}$ \\
\hline
\end{tabular}

Table 6: Popular E6 models - PyтHIA implementation. We used the notation: $s \equiv \sin \theta_{W}=$ $\sqrt{0.23}$.

- $Z_{N}^{\prime}$ is obtained in the $10+x \overline{5}$ model-line (last row in Table 4 ), for $x=2$ and $\gamma_{Z^{\prime}}=\frac{\sqrt{6}}{4} \sin \theta_{W}$

We finally note that Ref. [5] uses a different definition of $\theta: Z^{\prime}=Z_{\psi} \cos \theta-Z_{\chi} \sin \theta$. Working out the $V$ and $A$ couplings by using Eqn. (2.7) and Table 2 from [5], we obtain the same PyтнIA couplings as given in our Table 5. Using the definitions (i.e. $\theta$ values) of $Z_{\psi}^{\prime}$, $Z_{\chi}^{\prime}, Z_{\eta}^{\prime}$, and $Z_{I}^{\prime}$ given in Ref. [5], we find the couplings for $Z_{\psi}$ and $Z_{\eta}$ to be identical to the ones listed in our Table 6 , while the couplings for the $Z_{\chi}$ and $Z_{I}$ are equal to the negative of the corresponding values from Table 6 (which is equivalent to the transformation $\theta \rightarrow-\theta$ ). Figure 1 shows a cartoon with several definitions of the E6 angle $\theta$. 


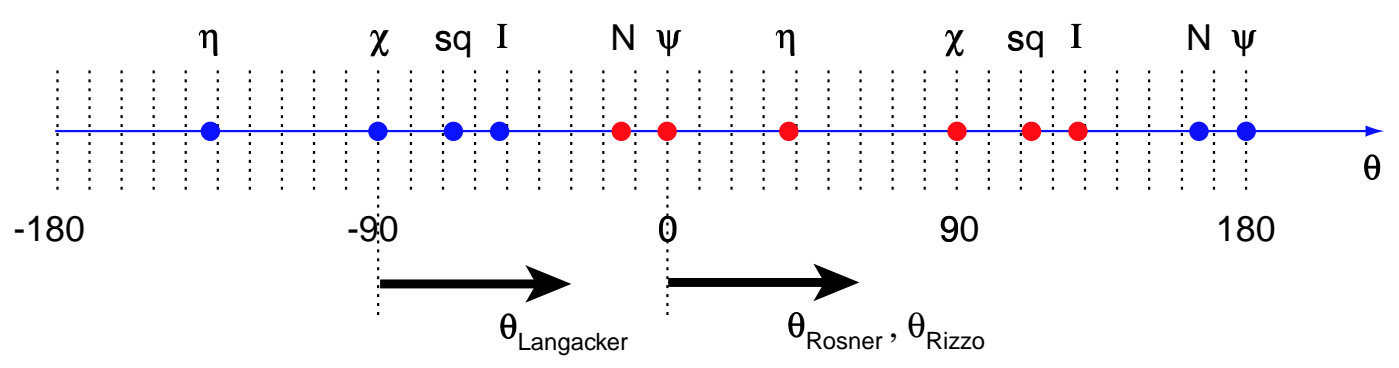

Figure 1: Several definitions of the E6 angle $\theta$.

\section{Conclusions}

In this document we briefly list several conventions used in the $Z^{\prime}$ literature, and give the PYTHIA implementation for the sequential $Z^{\prime}$, the model-lines of Ref. [2], and the popular E6 models. We hope this will serve as a useful first guide for the $Z^{\prime} /$ PYTHIA beginner. We thank Marcela Carena, Bogdan Dobrescu, Tim Tait, and Muge Karagoz for useful discussions.

\section{References}

[1] T. Sjöstrand et al., Comput. Phys. Commun. 135, 238 (2001). The discussion is valid for PYTHIA versions $6.2 x x-6.3 x x$.

[2] M. Carena, A. Daleo, B. Dobrescu, T. Tait, Phys. Rev. D 70, 093009 (2004).

[3] F. Halzen, A.D. Martin, "Quarks and Leptons: An Introductory Course in Modern Particle Physics”, John Wiley \& Sons, Inc., 1984.

[4] J.L. Rosner, Phys. Rev. D 35, 2244 (1987).

[5] J.L. Hewett and T.G. Rizzo, Phys. Rept.183, 193 (1989).

[6] The $Z_{N}^{\prime}$ and the secluded $Z_{s q}^{\prime}$ E6 models are taken from: J.Kang and P. Langacker, Phys. Rev. D 71, 035014 (2005). 